Rapport - Société canadienne d'histoire de l'Église catholique

\title{
Un projet de journal ecclésiastique de Mgr Lartigue
}

\section{Thomas-M. Charland}

Volume 24, 1956-1957

URI : https://id.erudit.org/iderudit/1007432ar

DOI : https://doi.org/10.7202/1007432ar

Aller au sommaire du numéro

Éditeur(s)

La Société canadienne d'histoire de l'Église catholique

ISSN

0318-6148 (imprimé)

1927-7075 (numérique)

Découvrir la revue

Citer cet article

Charland, T.-M. (1956). Un projet de journal ecclésiastique de Mgr Lartigue. Rapport - Société canadienne d'histoire de l'Église catholique, 24, 39-53.

https://doi.org/10.7202/1007432ar

Tous droits réservés @ La Société canadienne d'histoire de l'Église catholique, 1958
Ce document est protégé par la loi sur le droit d'auteur. L’utilisation des services d'Érudit (y compris la reproduction) est assujettie à sa politique d'utilisation que vous pouvez consulter en ligne.

https://apropos.erudit.org/fr/usagers/politique-dutilisation/ 


\section{Un projet de journal ecclésiastique de Mgr Lartigue}

L'idée de fonder un journal ecclésiastique au Canada français vint d'un prêtre d'ascendance irlandaise, l'abbé Thomas Maguire, alors qu'il était curé de Saint-Michel de Bellechasse (1806-1827). Une dizaine d'années durant, il multiplia les démarches pour la faire aboutir, mais toujours sans succès. Un moment, vers 1824, il conçut quelque espoir. A la prière de quelques amis et avec l'agrément de son supérieur hiérarchique, il présenta un projet concret aux curés des paroisses situées en bordure du fleuve, entre Québec et Montréal. Il ne recueillit guère plus d'une centaine de souscriptions. Ce piètre résultat abattit son courage et, l'année suivante, la mort de $\mathrm{M}^{\mathrm{gr}}$ Plessis (4 décembre 1825) vint mettre un terme à ses projets.

Il avait eu l'appui de l'évêque de Québec ${ }^{1}$. C'est celui du clergé qui lui avait manqué. Voici à quelles causes il attribuait son échec : une résistance ouverte de la part de quelques individus, pour qui le journal projeté serait un foyer de discorde, une pénurie de talents dans le corps ecclésiastique, chez la plupart des curés une ignorance complète des machinations même les plus ouvertes de leurs ennemis, chez quelquesuns de l'apathie, chez d'autres de l'indifférence pour tout ce qui se passe au-delà de leur horizon étroit, et chez un grand nombre une malheureuse parcimonie qui influe sur toute leur conduite et les empêche de venir au secours de la religion foulée aux pieds.

Fallait-il abandonner tout espoir de fonder un journal pour protéger les droits de la religion ? Oui, pensait l'abbé Maguire, aussi longtemps que le projet resterait entre les mains d'humbles individus sans crédit, sans appui et surtout sans ressources pécuniaires. Mais si le projet émanait de plus haut, et si les supérieurs ecclésiastiques unissaient leurs efforts en faveur d'un tel établissement, il serait alors possible d'effectuer une révolution à cet égard dans les sentiments du clergé.

C'est dans une lettre à $\mathbf{M}^{\text {gr }}$ Jean-Jacques Lartigue que M. Maguire exposait ainsi les causes de son échec et les conditions de succès pour la reprise de son projet ${ }^{2}$. $\mathbf{M}^{\mathrm{gr}}$ Lartigue s'était montré favorable à l'idée de M. Maguire. Il allait désormais s'employer avec une ténacité remarquable à en hâter la réalisation. S'il n'en avait tenu qu'à lui, l'affaire aurait réussi. Mais il n'était pas libre d'agir à sa guise : il lui fallait l'agrément de l'évêque de Québec, car il était non pas évêque

1 \& $\mathrm{M}^{\mathrm{gr}}$ le grand Plessis avait été pour un pareil établissement en 1818 », écrit l'abbé Jacques Paquin, dans ses Mémoires sur lEglise du Canada (manuscrit aux Archives Canadiennes), p. 989.

2 M. Maguire à $\mathrm{M}^{\mathrm{gr}}$ Lartigue, 5 mai 1827 (Archives de l'archevêché de Montréal, cartable-MM. Maguire et Tabeau). 
de Montréal mais évêque à Montréal, c'est-à-dire chargé du gouvernement spirituel du district de Montréal sous la dépendance de l'évêque de Québec. Et c'est le refus obstiné de l'évêque de Québec, $\mathbf{M}^{\text {gr }}$ Panet, qui lui fit abandonner finalement la partie.

J'ai dit que $M^{\mathrm{gr}}$ Lartigue s'était montré favorable au projet de M. Maguire. En effet, à l'automne de 1824, il offrit à $\mathbf{M}^{\mathrm{gr}}$ Plessis un prêtre de son district, M. Jacques de Lamothe, pour le poste de chapelain des Ursulines, aux Trois-Rivières, " seulement dans le cas où on le jugerait propre à rédiger le Journal ecclésiastique projeté ${ }^{3}$ ». Il était question de placer M. de Lamothe à la direction du Collège de SaintHyacinthe; mais $\mathbf{M}^{\mathrm{gr}}$ Lartigue estimait l'utilité d'un bon journal plus grande et plus générale que celle d'un collège particulier. $\mathrm{M}^{\mathrm{gr}}$ Plessis ne répondit pas à cette offre, qui sans doute ne lui agréait pas; et M. de Lamothe fut envoyé à Saint-Hyacinthe, où il ne demandait pas mieux que d'aller.

$M^{\text {gr }}$ Plessis mort, $M^{\text {gr }}$ Lartigue revint à la charge auprès de son successeur, $\mathrm{M}^{\mathrm{gr}}$ Panet, et profita de toutes les occasions pour ramener sur le tapis le projet du journal ecclésiastique.

En février 1827, il apprit qu'un journal anglican ${ }^{4}$ était sur le point de paraître dans sa ville de Montréal. Connaissant le zèle du nouvel évêque anglican, Charles-James Stewart, il s'attendait de le voir s'immiscer dans les affaires de religion et d'éducation. Il s'empressa de proposer à $\mathrm{M}^{\mathrm{gr}}$ Panet de préparer un antidote : le journal ecclésiastique dont il est question depuis quelques années. L'homme tout désigné pour en prendre la direction est le curé de Saint-Michel, l'abbé Maguire, celui-là même qui s'est déjà remué en sa faveur, et qui vient de se signaler comme polémiste plein de verve dans ses Observations d'un catholique sur l'histoire de l'honorable $W m$ Smith ${ }^{5}$. On aurait le concours de tous les membres du clergé, à qui il suffirait de faire sentir l'absolue nécessité de l'entreprise, et aussi la coopération de bon nombre de laïcs ${ }^{6}$. $M^{\text {gr }}$ Panet ne se laissa pas émouvoir, et se contenta de répondre : Attendons le premier numéro du journal ecclésiastique protestant qui va paraître et alors on verra ce qu'il y aura à faire ?. . De fait, la feuille ne parut pas dangereuse. Qu'à cela ne tienne, rétorqua $\mathrm{M}^{\mathrm{gr}}$ Lartigue, elle le deviendra infailliblement, et alors il est urgent de se préparer à en publier une bonne, qu'on pourra lui opposer dans la suite ${ }^{8}$.

$M^{g r}$ Lartigue venait de recevoir la réponse de $M$. Maguire, à qui il avait demandé son opinion sur la résurrection de son projet. M. Ma. guire faisait les suggestions suivantes sur la marche à suivre. Il faudrait

$3 \mathrm{M}^{\mathrm{gr}}$ Lartigue à $\mathrm{M}^{\mathrm{gr}}$ Plessis, 23 octobre 1824 (AAM, registre des lettres 3:118).

4 The Christian Sentinel.

5 Parues dans le Canadien de Québec, 11 janvier 1827, sous le pseudonyme de Vindex, et reproduites en une brochure de treize pages.

$6 \mathrm{M}^{\mathrm{gr}}$ Lartigue à $\mathrm{M}^{\mathrm{gr}}$ Panet (AAM, registre des lettres 4:223).

$7 \mathrm{M}^{\mathrm{gr}}$ Panet à $\mathrm{M}^{\mathrm{gr}}$ Lartigue, 22 février 1827 (Archives de l'archevêché de Québec, registre des lettres 13:132).

$8 \mathrm{M}^{\mathrm{gr}}$ Lartigue à $\mathrm{M}^{\mathrm{gr}}$ Panet, 12 mai 1827 ( $\mathrm{AAM}$, registre des lettres 4:240). 
que les supérieurs adressent un prospectus du journal, en l'accompagnant d'une lettre privée et pressante, à tout le clergé du diocèse, et qu'on confie ce prospectus et cette lettre à quelques membres influents du clergé pour qu'ils les fassent valoir auprès des individus; qu'on obtienne des souscriptions pour trois ans au moins, et que le montant en soit doublé et même triplé par les curés à l'aise; qu'on se procure des secours de la Maison de Saint-Sulpice et que, au besoin, l'Ordinaire emploie quelques componendes ou autres argents à sa disposition pour achever de couvrir les frais du journal jusqu'à ce qu'il puisse se soutenir par lui-même. Le journal devrait être publié à Montréal, qui est plus central pour les communications et plus à proximité des talents. Resterait la difficulté la plus sérieuse, celle de trouver un éditeur. Ce devrait être naturellement un ecclésiastique. Mais comme il n'y en a aucun qui soit vraiment qualifié pour cette tâche, et que ceux qui pourraient s'y consacrer ne le voudraient pas ou ne seraient pas libres de le faire, il n'y a pas d'autre alternative que d'en charger un laïque, qui serait sous la surveillance d'un ou deux ecclésiastiques nommés par les supérieurs ${ }^{9}$.

L'année suivante (1828), $\mathrm{M}^{\mathrm{gr}}$ Lartigue constatait avec honte que les Eglises anglicane et presbytérienne avaient chacune leur journal au pays, alors que l'Eglise catholique n'y avait pas encore le sien. a Nous avons un besoin urgent de la presse pour venger avec vigueur et prudence la Religion et nos droits attaqués de tous côtés; et aucune des sectes protestantes ne pourrait se plaindre parce qu'elles nous ont harassés les premières. " $\mathrm{M}^{\mathrm{gr}}$ Panet n'aurait qu'à recommander le projet au clergé du diocèse et tout s'arrangerait en peu de temps. M. Maguire assumerait la direction de la feuille. Il n'est pas à sa place comme directeur du Collège de Saint-Hyacinthe. "Son genre serait d'étudier et d'écrire, ce qu'il pourrait faire avec avantage, s'il était dirigé par quelqu'un qui modérât la chaleur de sa tête et la vivacité de son esprit... Si l'on jugeait que $M$. Maguire fût bien à Montréal pour la direction d'un pareil journal, je le recevrais volontiers à Saint-Jacques : il y serait exempt de confessions, pourvu qu'il voulût prêcher en anglais, et même sans cela s'il le fallait ${ }^{10}$,

$\mathrm{M}^{\mathrm{gr}}$ Lartigue ne reçut pas de réponse à ses propositions. Au bout de cinq mois, il écrivit de nouveau à $M^{\text {gr }}$ Panet, disant que le projet de journal ecclésiastique lui tenait toujours fort à cœur, et faisant valoir les deux mêmes arguments déjà invoqués : d'une part, la nécessité qui s'imposait de plus en plus de fonder ce journal, de l'autre, la possibilité d'y arriver sans trop de difficultés ${ }^{11}$. Cette fois $\mathbf{M}^{\mathrm{gr}}$ Panet répondit, mais négativement. Il croyait qu'un tel journal deviendrait nécessaire dans quelques années, mais ne l'était pas pour le moment. Il ne voyait d'ailleurs personne qui fût capable de le rédiger, et il craignait que les frais n'en retombent que sur quelques-uns. Il ajoutait, et c'est sans

M. Maguire à $\mathrm{M}^{\mathrm{gr}}$ Lartigue, 5 mai 1827 (AAM, MM. Maguire et Tabeau).

$M^{\mathrm{gr}}$ Lartigue à $\mathrm{M}^{\mathrm{gr}}$ Panet, 25 juillet 1828 (AAM, registre des lettres 4:361). $\mathrm{M}^{\mathrm{gr}}$ Lartigue à $\mathrm{M}^{\mathrm{gr}}$ Panet, 30 novembre 1828 (AAM, registre des lettres 4:437). 
doute là la vraie raison de son refus de s'engager dans cette entreprise : - Quant à moi qui m'épuise pour bâtir le Séminaire de Nicolet, je n'oserais me charger de faire aller le papier en question ${ }^{12}$. $\mathbf{M}^{\mathrm{gr}}$ Panet s'était, en effet, mis en frais de construire le nouvel édifice du Séminaire de Nicolet, et avait lancé une souscription parmi les membres du clergé $^{13}$. Le parachèvement de cette construction, grandiose pour l'époque, allait absorber toutes les économies de l'évêque de Québec, et aussi toutes ses pensées.

Entre-temps, éclata en France la révolution de juillet 1830. Belle occasion pour $\mathrm{M}^{\mathrm{gr}}$ Lartigue de reprendre son projet et de faire valoir auprès de $\mathbf{M}^{\mathrm{gr}}$ Panet de nouvelles raisons en faveur de sa réalisation. "Voici, lui écrivit-il, le moment le plus pressant peut-être qu'il y ait eu pour la Province, d'y établir sur un bon pied un Journal ecclésiastique, afin de contrecarrer les diatribes révolutionnaires qui vont retentir, et qui ont déjà commencé à paraître dans tous nos papiers... Si nous laissons le temps aux journaux démocrates, qui pullulent en ce pays, de verser le poison de leurs erreurs parmi nos peuples sans leur présenter l'antidote, il ne sera plus temps d'essayer à ramener au bon chemin l'esprit public lorsqu'il aura été une fois faussé ${ }^{14}$. ,

"C'est sans doute une belle chose de bâtir une maison d'éducation religieuse, mais former l'esprit public est encore plus pressant. Est-ce que le clergé et les honnêtes gens ne pourraient pas se cotiser au plus tôt pour soutenir une feuille hebdomadaire qui, en défendant la religion selon l'occurrence, opposerait les vrais principes aux déclamations des démagogues? Assurément je suis pauvre, mais j'y mettrais volontiers tout mon avoir. Les catholiques du Haut-Canada, qui ne sont qu'une poignée à côté de nous, ont fondé un journal, The Catholic ${ }^{15}$, pour défendre la Religion. N'est-il pas honteux que nous restions ici les bras croisés à les regarder faire? Il s'agirait d'une dépense annuelle de 300 louis. On trouverait à Montréal un imprimeur à bon compte. Le rédacteur serait M. Maguire, pourvu qu'il voulût se faire aider et un peu diriger. Mais alors il faudrait prendre une décision avant la fin des vacances, car M. Maguire, de retour d'une mission à Rome, attend à Québec avant de reprendre son poste de directeur au Collège de SaintHyacinthe ${ }^{16}$.,

En même temps, $M^{g r}$ Lartigue écrivit à $M$. Maguire, le priant de stimuler $\mathbf{M}^{\mathrm{gr}}$ Panet et d'aviser avec lui aux moyens propres à réaliser le projet. Il était prêt, quant à lui, à offrir sa maison à qui voudrait se charger d'une si bonne cuvre ${ }^{17}$. Le vieil évêque de Québec persista dans son refus.

$12 M^{\mathrm{gr}}$ Panet à $\mathrm{M}^{\mathrm{gr}}$ Lartigue, 27 décembre 1828 (AAQ, registre des lettres 13:504). 13 Circulaire du 31 octobre 1826 (Mandements des Evêques de Québec 3:224-226).

$14 M^{\mathrm{gr}}$ Lartigue à $\mathrm{M}^{\mathrm{gr}}$ Panet, 11 septembre 1830 (AAM, registre des lettres 5:306).

15 R. F. Cronin, Early Catholic Journalism in Canada (The Canadian Catholic Historical Association, Report 1935-1936: 33).

18 C.-P. Choquette, Histoire du Séminaire de Saint-Hyacinthe, 1: 95-98.

$17 M^{\mathrm{gr}}$ Lartigue à $\cdot \mathrm{M}$. Maguire, 17 sept. 1830 (AAM, registre des lettres 5:313). 
Puis voici que, au début de 1831 , se presenta l'affaire des notables. Dans la plupart des paroisses du Bas-Canada, les assemblées de fabrique pour l'élection des marguilliers et la reddition des comptes se composaient uniquement des marguilliers anciens et nouveaux, en conformité avec des règlements qui remontaient au temps de $\mathrm{M}^{\mathrm{gr}}$ de Laval. Dans quelques paroisses, l'usage s'était introduit d'admettre en plus un certain nombre de paroissiens reconnus comme notables. En 1830, on voulut généraliser et légaliser cet usage. Des pétitions en ce sens furent adressées à la Chambre par des groupes de la région de Saint-Hyacinthe. Et, au début de 1831, le député du comté de Nicolet, Louis Bourdages, présenta un bill en faveur de l'admission des notables en général. C'était un empiètement manifeste du pouvoir civil sur les droits de l'Eglise. La session fut prorogée avant que le bill ait subi sa troisième lecture. La Chambre s'était prononcée contre le bill tel que présenté. Mais on prévoyait que Bourdages reviendrait à la session d'automne avec un bill amendé. Il y revint en effet, mais pour réclamer cette fois l'admission des propriétaires, c'est-à-dire d'à peu près tous les habitants. La Chambre passa outre à une pétition des évêques et du clergé demandant le rejet de toute mesure de ce genre, et le bill Bourdages fut adopté par trente voix contre dix-neuf. Il échoua toutefois au Conseil législatif, à grande majorité protestante, qui le renvoya à six mois, c'est-à-dire aux calendes grecques ${ }^{18}$.

La discussion du bill des notables, à la Chambre et dans les journaux, donna lieu à de virulentes diatribes contre l'influence du clergé. Ce dernier se défendit. Mais il n'y avait que la Gazette de Québec et le Canadien qui voulussent accepter ses répliques. Aussi $\mathbf{M}^{\mathrm{gr}}$ Lartigue, en s'ouvrant à $\mathbf{M}^{\mathrm{gr}}$ Panet de son dessein d'écrire dans l'un ou l'autre de ces journaux une série d'articles contre les discours faits en Chambre par le parti des notables ${ }^{19}$, ne manquait pas d'ajouter : - Les difficultés que nous éprouvons quand il s'agit d'imprimer quelque chose qui concerne les intérêts du clergé et de l'Eglise, montrent de plus en plus la nécessité qu'il y a, même depuis longtemps, d'avoir une presse et un Journal ecclésiastique, que je serai toujours prêt, ainsi que l'immense majorité du clergé, à favoriser, dès que l'autorité supérieure voudra bien s'y prêter : plus on retarde cette mesure devenue indispensable par les circonstances, plus nous nous faisons tort ${ }^{20}$. " Et quelques jours plus tard, en lui soumettant son premier article : « Si vous vous mettiez à la tête de ce projet, il serait prêt à marcher en peu de temps et sans qu'il en coutât que bien peu de chose à l'Evêque, parce que l'entreprise se ferait pas souscription ${ }^{21}$. ,

$M^{27}$ Panet jugea l'article plus capable d'irriter les membres de la Chambre que de faire impression dans le public. Il le renvoya à son auteur en lui demandant de ne pas le publier sans en retrancher \& une

15 Thomas Chapais, Cours d'Histoire du Canada, III (Québec, 1921), p. 245-261.

19 Sous le pseudonyme de Un Catholique, $\mathrm{M}^{\mathrm{gr}}$ Lartigue s'en était déjà pris aux discours de son cousin, Louis-Joseph Papineau, dans La Minerve du 5 mai 1831.

$20 \mathbf{M}^{\mathrm{g}}$ Lartigue à $\mathbf{M}^{\mathrm{gr}}$ Panet, 16 déc. 1831 (AAM, registre des lettres 6:174).

21 Le même au même, 23 déc. 1831 (ibid., 6:182). 
attaque contre Messieurs Duval et Mondelet, qui ne peut que nous faire perdre l'appui de ces jeunes messieurs auxquels on ne peut reprocher que de la légèreté ${ }^{22}$ ». Quant au projet du journal ecclésiastique, il refusait de participer à sa réalisation et motivait cette fois son refus par une appréhension : " Je craindrais, dit-il, qu'il ne nous mît continuellement aux prises avec les ennemis du clergé ${ }^{23}$.

$M^{\mathrm{gr}}$ Lartigue fut mécontent du reproche de n'avoir pas ménagé les membres de la Chambre. Il le fut plus encore du refus d'encourager le journal ecclésiastique, "parce que, disait-il, c'est nous priver du seul remède qui, après Dieu, pourrait guérir nos maux à l'époque actuelle; mais je ne me reprocherai pas de n'avoir pas fait mon possible pour cet établissement ${ }^{24}$. Il ne renonça pas à tout espoir de gagner $\mathbf{M}^{\text {gr }}$ Panet. Comme il prévoyait que le bill des notables reviendrait devant l'Assemblée législative, il proposa à M. Pierre-Flavien Turgeon, procureur du Séminaire de Québec, une réédition à cinq ou six cents exemplaires de la brochure de l'avocat André-Rémi Hamel, La question des fabriques, par un Ami de l'Ordre, pour la répandre dans les campagnes, "car cet ouvrage est plus à la portée du peuple que notre Mémoire $^{25}$; mais forcez principalement sur l'établissement d'un Journal ecclésiastique ${ }^{26} \gg$.

Dans sa lettre du 23 décembre 1831 accompagnant l'envoi de son premier article, $M^{\mathrm{gr}}$ Lartigue avait prévenu $\mathrm{M}^{\mathrm{gr}}$ Panet : " Les prêtres de mon district me tourmentent en faveur de ce projet, et voulant absolument l'effectuer, je ne vois pas comment je pourrais m'exempter de me joindre à eux, si l'on ne fait rien à Québec là-dessus : étant à leur tête, je pourrais du moins les diriger, modérer la fougue de quelques-uns, et donner quelque poids à leur entreprise, qu'il serait pourtant triste de ne pas voir générale pour tout le clergé du diocèse. " Or, le 29 du même mois, neuf prêtres du district de Montréal tinrent une assemblée au presbytère de Sainte-Geneviève de Pierrefonds, sous la présidence de M. Raizenne, curé de Saint-Benoît. Ils se prononcèrent unanimement pour la fondation d'un journal ecclésiastique, qui s'appellerait L'Ami du clergé et du peuple canadien. Ce journal servirait de voie de communication entre les membres du clergé, dispersés sur un vaste territoire, et entre le clergé et la masse des fidèles, de tribune pour défendre les droits de l'Eglise et d'organe pour éclairer le peuple sur ses vrais intérêts. Il ne serait en aucune circonstance l'écho d'un parti et se garderait de soulever ou de réveiller, sans provocation, aucune polémique ecclésiastique ou politique; mais il affirmerait sa loyauté en étant le soutien vrai et impartial de la métropole et de la constitution coloniale du pays. Il

Le 13 décembre 1831, $M^{\text {gr }}$ Lartigue écrivait à $M^{\text {gr }}$ Signay : « Notre avocat Duval s'est aussi distingué par la mauvaise défense qu'il a faite pour nous que par la motion qu'il vient de faire qu'on ôte les registres des paroisses à nos curés 》 (AAM, registre des lettres $6: 167$ ).

$23 \mathrm{M}^{\mathrm{gr}}$ Panet à $\mathrm{M}^{\mathrm{gr}}$ Lartigue, 28 déc. 1831 (AAQ, registre des lettres 14:542).

$24 \mathrm{M}^{\mathrm{gr}}$ Lartigue à $\mathrm{M}^{\mathrm{gr}}$ Panet, 31 déc. 1831 (AAM, registre des lettres 6:188).

25 Il s'agit du mémoire accompagnant la requête du clergé à la Chambre (note de l'auteur).

$26 \mathrm{M}^{\mathrm{br}}$ Lartigue à M. P.F. Turgeon, 3 janvier 1832 (AAM, reg. des lettres 6:190). 
fut résolu d'ouvrir immédiatement une souscription, de solliciter l'appui des évêques de Québec et de Montréal, et de prier les grands vicaires d'organiser dans leurs quartiers des comités qui nommeraient chacun un député autorisé à prendre avec les supérieurs hiérarchiques les derniers arrangements pour la nomination des rédacteurs, la diffusion du journal et autres affaires.

Une autre réunion de prêtres devait avoir lieu le 3 janvier suivant au presbytère de Saint-Charles de la Rivière-Chambly pour traiter de l'affaire des notables. Le secrétaire de l'assemblée de Sainte-Geneviève, l'abbé Jacques Paquin, y fut député pour savoir l'opinion de ses membres sur le projet du journal ecclésiastique. On y adopta en entier les résolutions de Sainte-Geneviève, et on constitua sur le champ le comité de la Rivière-Chambly. On fut aussi d'avis qu'il fallait acquérir au plus tôt une presse, dont la propriété pourrait être transférée à la Caisse ecclésiastique de Saint-Michel ${ }^{27}$.

Le 7 janvier, $\mathbf{M}^{\mathrm{gr}}$ Lartigue annonçait à $\mathrm{M}^{\mathrm{gr}}$ Panet qu'un grand nombre de curés de son district s'étaient organisés en comités et qu'une souscription ouverte en faveur du journal ecclésiastique avait eu un grand succès. Il revenait sur la nécessité de ce journal pour réussir à diriger l'opinion publique. Et il insistait encore pour que l'évêque de Québec se mît à la tête de l'entreprise : les prêtres paraissent résolus à lui en remettre l'entière direction; mais s'il refuse d'y prendre part, elle se fera sans lui, et alors qui sait si, dans quelques circonstances semblables à celles où le clergé s'est déjà trouvé, la direction du journal ne sera pas en désaccord avec les sentiments de l'évêque ? $\mathbf{M}^{\mathrm{gr}}$ Panet ne céda pas devant cet argument. Il répondit à $M^{\text {gr }}$ Lartigue : « Quant à l'établissement d'un journal ecclésiastique que vous paraissez désirer avec tant d'ardeur, je vous ai déjà marqué ce que j'en pensais. Je ne puis me résoudre à me mettre à la tête de l'entreprise, dans la crainte qu'elle ne soit par la suite une source de déboires pour les Evêques. Vous pouvez prendre à cet égard telle démarche qu'il vous plaira; pour moi permettez que je me tienne à l'écart ${ }^{28}$."

M. Antoine Tabeau, grand vicaire de $\mathbf{M}^{\mathrm{gr}}$ Lartigue, avait été nommé agent de liaison entre les différents comités. En cette qualité, il adressa copie des résolutions de Sainte-Geneviève et de Saint-Charles à $\mathrm{M}^{\mathrm{gr}}$ Panet et à son coadjuteur, $\mathrm{M}^{\mathrm{gr}}$ Signay, ainsi qu'aux grands vicaires du diocèse : MM. Jérome Demers et Thomas Maguire, du Séminaire de Québec, Pierre Viau, curé de la Rivière-Ouelle, et Louis-Marie Cadieux, curé des Trois-Rivières.

\section{A $\mathrm{M}^{\text {gr }}$ Panet, il écrivit (9 janvier 1832) :}

Fermement attachés aux principes de la subordination hiérarchique, nous désirons tous que les Evêques, comme premiors pasteurs, et conséquemment plus intéressés que tout autre à préserver de toute contagion le troupeau de Jésus-Christ, soient nos guides et nos soutiens dans cette 
entreprise, et nous les prions humblement d'appuyer de leur sanction les efforts que fait le clergé pour défendre cette Religion sainte, dont nous sommes les ministres, contre les viles attaques auxquelles elle est maintenant en butte. Daignez, Monseigneur, approuver la bonne volonté de vos prêtres, qui est ici générale; et, sans aucune dépense pécuniaire de votre part, vous aurez bientôt à votre disposition une arme aussi puissante que nécessaire, celle de la presse, pour combattre les ennemis qui s'élèvent de toutes parts contre le Seigneur et son Christ 29.

\section{$\mathrm{M}^{\mathrm{gr}}$ Panet répondit (16 janvier) :}

Je suis persuadé que les meilleurs motifs ont dirigé ces messieurs dans leur démarche; mais leur projet, il me semble, a besoin d'être mûri, avant que les Evêques se déterminent à se mettre à la tête de l'entreprise et à se rendre pour ainsi dire responsables de tous les écrits qui paraîtront dans le journal en question. On ne trouvera pas mauvais, je l'espère, que je sois neutre dans cette affaire, du moins jusqu'à ce que je voye clairement comment sera dirigé ce journal, dont le but est sans doute de favoriser la cause de la Religion, mais qui pourrait bien aussi être cause de certains partis auxquels je ne voudrais pas donner occasion 30 .

Même hésitation et même crainte chez le coadjuteur, $\mathrm{M}^{\mathrm{gr}}$ Signay. La réponse de ce dernier est beaucoup plus explicite que celle de $\mathrm{M}^{\mathrm{gr}}$ Panet, qui s'en est d'ailleurs peut-être inspiré ${ }^{31}$. Aussi me permettrai-je de la citer en son entier :

J'ai reçu votre lettre avec les résolutions y contenues, et je vous remercie en vous observant que l'objet qui y est mentionné est d'une trop grande importance pour qu'on puisse y répondre définitivement dans un si court espace de temps.

Tout le monde sent l'avantage d'une Imprimerie du clergé, sagement dirigée et destinée à répandre au milieu des peuples confiés à ses soins, des écrits marqués au coin de la charité et de la modération, propres à les instruire et à leur faire sentir qu'il est essentiellement attaché à leur religion et aux sages et précieuses institutions transmises par leurs pères, etc. Mais avant que les supérieurs puissent répondre décidément sur la nouvelle et intéressante entreprise mentionnée dans les dites résolutions, on ne doit pas trouver mauvais de leur part, qu'ils désirent avoir des connaissances ultérieures des moyens sur lesquels on compte pour la soutenir, ne postea quam, etc., et de la qualification des Directeurs et examinateurs des publications théologiques et politiques (car il ne paraît pas que ces dernières soient retranchées du Prospectus). Il convient aussi que les dits Supérieurs sachent et examinent jusqu'à quel point, en approuvant spécialement cette mesure, ils pourraient être concernés dans ses effets, et par suite, responsables à l'égard de ce qui pourrait s'échapper d'inconsidéré et de repréhensible dans les diverses publications adressées à cette Imprimerie, vu que chacun, en vertu de sa souscription, se croira suffisamment autorisé à insérer les productions de sa plume et "selon l'abondance de son sens ", quelles qu'elles soient. On ne doit pas non plus trouver mauvais qu'ils puissent être bien assurés que, dans les publications qui touchent l'intérêt ou la défense de la Religion, ou les prérogatives du clergé, on évitera ce désordre si commun,

29 AAQ, Vicaires Généraux 5:109.

30 AAQ, registre des lettres 14:562.

31 Ce qui me le fait croire, c'est ce passage d'une lettre de $M^{\text {gr }}$ Panet à $M^{\mathbf{g}^{\mathrm{r}}}$ Lartigue (6 mars 1832) : « Au reste, vous pouvez sonder les dispositions de $\mathbf{M}^{\mathrm{gr}}$ le Coadjuteur qui a correspondu à ce sujet avec M. Tabeau 》(AAQ, registre des lettres 15:34). 
et que la charité réprouve, je veux dire ces personnalités aigres, ces déclarations diffamatoires, et par conséquent si proches du libelle; en un mot toute insertion propre à provoquer une animosité de représaille, dont on ne connait plus le terme, et qui tend à faire émettre à la portée des faibles et des ignorants, tout ce qu'on peut recueillir d'humiliant pour le clergé, et capable de produire de mauvaises, et quelquefois ineffaçables impressions dans les esprits.

J'apprécie les observations par lesquelles vous terminez votre lettre, mais $j$ 'espère que vous ne regarderez pas avec indifférence celles que j'émets dans la présente. Je m'y crois d'autant plus autorisé que l'expérience apprend que, nonobstant les représentations sages et motivées de personnes éclairées et prévoyantes, il se trouvera certains écrivains qui, dans l'ardeur et l'envie dont ils sont animés de mettre les productions de leur plume au jour, passent sur toute considération, et, toujours dans la prétendue idée de faire le bien, sont disposés à insérer dans les papiers publics des tirades quelquefois aussi ridicules qu'anticharitables, dont ils ne veulent pas même examiner les suites et les inconvénients, je pourrais ajouter les dommages auxquels ils s'exposent, ainsi que ceux de la cause qu'ils prétendent défendre. Qu'on se donne la peine d'entendre là-dessus les Editeurs même les plus coulants, on verra combien de morceaux de la description de ceux dont je parle, ont été mis à l'index de leur Bureau. Qui sait même, d'après ce qui se dit, si on ne trouvera pas libelle dans certaines productions nouvellement insérées? Or, dans ce cas, à quel grave inconvénient ne s'expose pas un auteur de publication dont lui-même veut être le seul juge avant de l'émettre ? Au reste, en finissant, j'observerai que la lutte continuelle à laquelle sont exposés les journaux ecclésiastiques des Etats voisins de notre province - celui de Boston, Jésuite, trop ardent pour la bonne cause, sans mesure et sans délicatesse pour ses adversaires, a entrainé la ruine d'un établissement catholique - nous instruit assez de celle qu'éprouvera vraisemblablement un journal canadien du même genre, et par conséquent de la nécessité d'aviser, avant d'y entrer, aux moyens assurés de pouvoir y résister: Quis rex, iturus committere bellum, etc. ${ }^{32}$.

Des grands vicaires, seul $M$. Viau approuva entièrement les résolutions de Sainte-Geneviève et de Saint-Charles. Avant d'agir conformément à ces résolutions, $M$. Cadieux voulut consulter $\mathrm{M}^{\mathrm{gr}}$ Panet. Ce dernier le laissa libre de faire ce que bon lui semblerait, en lui rappelant toutefois que le grand vicaire Demers n'avait pas cru convenable d'aller de l'avant alors que les évêques réservaient leur approbation ${ }^{33}$. Les réflexions que se permirent MM. Demers et Maguire sur le projet de journal, bien qu'elles plurent en grande partie à $M^{\mathrm{gr}}$ Lartigue, ne l'arrêtèrent pas dans ses démarches pour en hâter la réalisation.

M. Maguire fut prié par $\mathbf{M}^{\mathrm{gr}}$ Lartigue de sonder, par lui-même ou par l'intermédiaire de l'honorable Panet et de M. Hamel, les imprimeurs du Canadien de Québec, MM. Fréchette et Compagnie, pour savoir si et à quelles conditions ils seraient disposés à se transporter à Montréal avec leur presse et à faire de leur journal un journal ecclésiastique, soumis au contrôle exclusif du clergé, qui fournirait un rédacteur compétent. $M^{\mathrm{gr}}$ Lartigue était d'avis que le journal ne changeât même

32 M Signay à M. Tabeau, 16 janv. 1832 (AAQ, N.D. de Q., 1:93).

$33 \mathrm{M}^{\text {gr }}$ Panet à M. Cadieux, 16 janv. 1832 (AAQ, registre des lettres 14:562). 
pas de titre, pour que la chose fit moins d'éclat. Il voulait que, les arrangements conclus, les imprimeurs partissent de Québec sans souffler un mot à personne de leur engagement avec le clergé. "Et surtout, ajoutait-il, j'entends que mon nom soit mis à l'écart dans toute cette affaire $^{34}$. M. Maguire eut vite fait de constater que les divers engagements qu'ils avaient pris empêchaient les imprimeurs du Canadien d'accepter l'offre qui leur était faite.

$\mathrm{M}^{\mathrm{gr}}$ Lartigue songea alors à tenter une démarche semblable auprès de Ludger Duvernay, de la Minerve de Montréal. Il trouvait ce parti encore plus avantageux, mais il doutait fort de pouvoir gagner Duvernay, " enflé qu'il est de l'appui qu'il reçoit du maire du peuple dans son attaque contre le Conseil (législatif) ${ }^{35} \gg$. Cette fois, M. Maguire fit tenir à Duvernay, par l'intermédiaire d'un laïque intelligent, une note écrite d'une main étrangère, contenant les deux propositions suivantes : $1^{\circ} \mathrm{M}$. Duvernay serait-il disposé à prendre un engagement avec un nouvel éditeur pour la continuation de son journal sous un autre titre et sous le contrôle du nouvel éditeur en ce qui regarde la rédaction; $2^{\circ}$ s'il n'est pas libre de rompre son engagement avec la Minerve, pourrait-il imprimer un second journal de même format, sous le contrôle de l'éditeur? Duvernay ne voulut discuter ni l'une ni l'autre de ces propositions, sans savoir positivement de qui elles venaient. Il avait probablement eu vent du projet de $\mathrm{M}^{\mathrm{gr}}$ Lartigue, ainsi qu'en témoigne cette lettre de M. Maguire :

On dit que la Minerve est très répandue, et de plus qu'elle est fortement appuyée de tous les libéraux du pays. Il s'ensuivrait qu'il n'est pas au pouvoir du clergé d'acheter ce journal pour le changer de titre; surtout si les libéraux sont informés de ce projet, comme il est très possible qu'ils le soient; car un laïque de Montréal a écrit ces jours derniers à un membre de la Chambre qui est actuellement à Québec, que Votre Grandeur, aidée du clergé du district et de la ville de Montréal, était sur le point de publier une gazette. Et qui sait si l'on n'a pas donné l'éveil à $M$. Duvernay 36 ?

On se garda bien de poursuivre la démarche auprès de Duvernay. A quelque temps de là, on pouvait lire dans le même numéro de la Minerve (16 février 1832) un article signé S... où la révolution était hautement proclamée, et un autre de l'éditeur contenant des propos diffamatoires contre les quatre juges de la Cour du Banc du Roi à Québec. En signalant ces articles à l'attention de $\mathbf{M}^{\mathrm{gr}}$ Panet, $\mathbf{M}^{\mathrm{gr}}$ Lartigue ajoutait ces lignes où se devine une allusion au refus qu'il avait essuyé de la part de Duvernay :

Croyez-vous maintenant que si nous avions une presse indépendante, telle que projetée pour notre Journal ecclésiastique, elle ne serait pas nécessaire pour bâillonner cette canaille; car telle est la liberté de la presse comme ils l'entendent, qu'ils refusent d'imprimer ce qui est contraire à leurs opinions, et qu'ils veulent même en ôter les moyens.

$34 \mathrm{M}^{\mathrm{gr}}$ Lartigue à M. Maguire, 17 janv. 1832 (AAM, registre des lettres 6:204).

$35 M^{\text {gr }}$ Lartigue à M. Paquin, 25 janv. 1832 (Paquin, Mémoires, p. 987). Duvernay fut arrêté le 15 janvier, comparut devant le Conseil législatif et fut détenu en prison jusqu'à la fin de la session, 25 février.

36 M. Maguire à $M^{\text {gr }}$ Lartigue, 4 fév. 1832 (AAM, cartable Saint-Eustache). 
Pour moi, après avoir fait tous mes efforts, je me lave les mains par rapport aux résultats que j'en redoute; et je serais fâché qu'on pût dire par la suite que le clergé n'a pas voulu empêcher le mal quand il l'aurait pu ${ }^{37}$.

Il n'y avait plus qu'à se tourner vers la petite presse du curé Pigeon, de Laprairie. M. Paquin proposa à $\mathbf{M}^{\mathrm{g}^{\mathrm{g}}}$ Lartigue de la louer, en attendant les moyens de l'acquérir ${ }^{38}$. Mais avant d'aller plus loin, $\mathrm{M}^{\mathrm{gr}}$ Lartigue voulut consulter son supérieur hiérarchique.

On se rappelle que $\mathrm{M}^{\mathrm{gr}}$ Panet, tout en voulant se tenir à l'écart du projet de journal, avait permis à $\mathbf{M}^{\mathrm{gr}}$ Lartigue d'entreprendre toute démarche qu'il lui plairait pour sa réalisation. Cette solution ne satisfaisait qu'à demi $\mathbf{M}^{\mathrm{gr}}$ Lartigue. Il écrivit à $\mathbf{M}^{\mathrm{gr}}$ Panet :

Nous serons obligés de marcher sans vous dans l'établissement d'un Journal ecclésiastique; mais outre que le défaut d'unanimité qui, par la non-coopération de vous et de votre coadjuteur, paraîtra dans cette démarche, à laquelle se porte de grand cour tout le reste du clergé, même le Séminaire de Montréal, je suis fâché que les Evêques se privent volontairement d'une arme qui aurait pu leur assurer un grand pouvoir, au lieu qu'elle se tournera peut-être contre eux dans la suite : car une des conditions expresses du Prospectus de ce papier devait être, d'après le projet primordial, qu'il ne s'en imprimerait pas une ligne qui ne fût soumise à la censure de l'Evêque ou de ses grands vicaires ${ }^{39}$.

De son côté, $\mathrm{M}^{\mathrm{gr}}$ Panet n'était guère rassuré pour l'avenir. Il l'avouait à $\mathbf{M}^{\mathrm{gr}}$ Lartigue :

Vous allez entreprendre l'établissement d'un Journal ecclésiastique. Je m'en réjouirai, s'il est conduit avec cette modération et cette prudence qui devraient continuellement accompagner les écrits du clergé. N'en. verra-t-on pas quelquefois à ce journal des écrits remplis de violence et de personnalités? Si on ne les reçoit pas, l'auteur blessé enverra son article à un autre journal. On murmurera contre ceux qui dirigeront la nouvelle gazette, et de là une source de nouveaux mécontentements. Je crains que ce papier ne soit un sujet de discorde non seulement entre le clergé et nos libéraux, mais encore entre les différents membres du clergé. Je souhaite de tout mon cœur que ces misères n'arrivent pas $\mathbf{4 0}$.

Ces sombres appréhensions n'altérèrent pas la décision de $\mathrm{M}^{\mathrm{gr}} \mathrm{Lar}$ tigue d'aller de l'avant. Il voulut toutefois savoir d'une manière définitive si $\mathbf{M}^{\mathrm{gr}}$ Panet n'avait aucune objection à ce qu'il se mît luimême à la tête du projet, afin de le diriger pour le plus grand bien ${ }^{41}$. $\mathbf{M}^{\mathrm{gr}}$ Panet évita de se prononcer catégoriquement. Sa réponse nous révèle toutefois le fond de sa pensée. On y voit que l'évêque de Montréal n'était pas étranger à la cause de ses appréhensions.

Pour qu'un évêque, lui écrivit-il, puisse se mettre convenablement à la tête de l'établissement projeté d'un Journal ecclésiastique, il faut que ce journal soit conduit avec beaucoup de prudence et de modération; qu'il évite toute discussion propre à réveiller certaines dissensions dans le clergé; qu'il refuse tout écrit propre à censurer aucune des branches

$37 M^{\text {gr }}$ Lartigue à $M^{g r}$ Panet, 18 fév. 1832 (AAM, registre des lettres 6:227).

38 M. Paquin à Mer Lartigue, 11 fév. 1832 (AAM, cartable Saint-Eustache).

$39 M^{\mathrm{gr}}$ Lartigue à $\mathrm{M}^{\mathrm{gr}}$ Panet, 24 janv. 1832 (AAM, registre des lettres 6:209).

$40 \mathrm{M}^{\mathrm{gr}}$ Panet à $\mathrm{M}^{\mathrm{gr}}$ Lartigue, 28 janv. 1832 (AAQ, registre des lettres 15:4).

$41 \mathrm{M}^{\mathrm{gr}}$ Lartigue à $\mathrm{M}^{\mathrm{gr}}$ Panet, 11 fév. 1832 (AAM, registre des lettres 6:225). 
de la Législature; qu'il respecte les personnes ayant l'autorité. Sans cela il serait toujours aux prises avec quelques papiers, et dans ses démêlés viendraient des personnalités qui ne pourraient que le discréditer dans l'esprit du public. Je ne puis idonc répondre à la question que vous me faites à ce sujet sans savoir si ces conditions seront remplies. D'ailleurs ne connaissant point quelles sont les règles du Journal et quels en seront les directeurs, je ne puis guère former mon opinion sur le bien ou le mal qu'il pourra produire. Quand même il ne paraitrait rien dans ce journal sans avoir préalablement reçu votre approbation, n'aurais-je pas toujours lieu de craindre qu'il ne renfermât quelque chose de trop piquant, quelque personnalité injurieuse? Permettez-moi de vous le dire, Monseigneur, votre écrit signé idem me porte à croire que le papier projeté ne serait pas toujours conduit avec la modération convenable, si vous en étiez le directeur ${ }^{42}$.

L'écrit que vise ici $M^{\mathrm{gr}}$ Panet est un article paru dans la Gazette de Québec du 13 janvier 1832, où $\mathbf{M}^{\mathrm{gr}}$ Lartigue s'en prenait à l'ignorance religieuse de ceux qui avaient voté pour le bill des notables ${ }^{43}$. On y lisait :

Nous en avons encore un exemple tout récent (d'ignorance religieuse) dans l'écrit mignon du Sr. T, inséré dans la Gazette de M. Neilson le 13 de décembre : l'auteur, qui semble être d'une force théologique capable de prendre un Janséniste pour un Ebéniste *, ignore que le Jansénisme est une hérésie désespérante condamnée par l'Eglise, tandis que le Molinisme, qu'il a la bonhommie de lui opposer comme sa contradiction, n'est qu'un système sur la grâce, qu'on peut soutenir sans danger pour la foi.

* Un curé de Paris qui assistait à la mort un certain homme qui travaillait en bois d'ébène, mais dont on lui avait rendu la foi suspecte, s'avisa de lui demander s'il n'était point Janséniste : "Oh ! non, mon père, répondit naïvement l'ouvrier, car je suis Ebéniste. 》

Le sieur T... avait répliqué, dans la Gazette du 16 :

... j'aime mieux croire que c'est au nom de la théologie que vous avez le pouvoir de juger ainsi et supposer que vous êtes un grand théologien, car, dans mon ignorance, je ne puis le deviner, pas même à la savante et lumineuse définition que vous faites du Jansénisme et du Molinisme. Si vous désirez, monsieur, que vos écrits fassent quelque impression sur les honnêtes gens, abstenez-vous à l'avenir de débiter de pitoyables contes comme celui dans la note au bas de votre écrit, de semblables platitudes ne sont bonnes que pour la canaille.

Ce que $M^{g r}$ Panet entendait reprocher à $M^{\mathrm{gr}}$ Lartigue, ce n'est peut-être pas tant sa prise de bec avec le sieur T... que son attaque contre les membres de la Législature, car il voulait à tout prix ne pas s'aliéner davantage les adversaires du clergé et ne pas décourager la bonne volonté de ses partisans.

$\mathrm{M}^{\mathrm{gx}}$ Lartigue encaissa le reproche sans mot dire et s'appliqua à calmer les inquiétudes de $\mathbf{M}^{\mathrm{gr}}$ Panet. Il ne croyait pas nécessaire ni même utile qu'il prît lui-même la direction du journal projeté. Il préférait même que celui-ci fût imprimé à Québec, où se trouvaient des

$42 \mathrm{M}^{\mathrm{gr}}$ Panet à $\mathrm{M}^{\mathrm{gr}}$ Lartigue, 16 fév. 1832 (AAQ, registre des lettres 15:15).

43 L'insertion en avait été obtenue difficilement (M. Maguire à $\mathrm{M}^{\mathrm{gr}}$ Lartigue, 20 janv. 1832, AAQ, MM. Maguire et Tabeau). 
prêtres - les messieurs du Séminaire, M. Maguire entre autres - ayant la capacité et la modération voulues, et plus de temps que ceux de Montréal pour s'en occuper.

Je vous l'ai déjà dit et répété : mettez-vous seulement, avec votre coadjuteur, à la tête de l'entreprise, pour lui donner l'élan; et le clergé s'accordera à mettre à votre disposition la presse, s'il y en a une, à vous donner le choix de ceux qui rédigeront ou dirigeront l'ouvrage, à en laisser la surveillance à vous seul ou à votre coadjuteur; en sorte qu'il ne s'y imprime pas une ligne sans votre volonté, et que vous pourriez refuser toutes les pièces, même les miennes, qui vous seraient adressées, et qu'on ne trouverait pas convenables. Que vous faut-il de plus pour ne voir aucun danger dans cette entreprise ? Mais plutôt quel avantage pour la Religion, si l'Evêque avait un aussi puissant moyen pour former et maitriser l'opinion publique, et la faire tourner au profit de l'Eglise 44 !

Et pour vaincre la résistance de $\mathrm{M}^{\mathrm{gr}}$ Panet, $\mathrm{M}^{\mathrm{gr}}$ Lartigue tentait d'alarmer sa conscience : "Il ne sera plus temps d'y revenir, quand les mauvais papiers, qui seuls maintenant ont saisi cette puissance, auront bouleversé toutes les têtes; et vos successeurs auront à vous reprocher de n'avoir pas su profiter de l'occasion de sauver la foi et les bons principes en ce pays."

Le vieil évêque ne céda pas d'un pouce et réfuta tous les arguments de $\mathrm{M}^{\mathrm{gr}}$ Lartigue. Aucun des prêtres du Séminaire de Québec n'a plus de temps qu'il lui en faut pour vaquer à ses occupations, sans excepter M. Maguire, qui d'ailleurs décline absolument l'emploi proposé. Le sentiment du clergé est partagé : un grand nombre de prêtres rejettent le journal annoncé par les résolutions de Sainte-Geneviève; d'autres le voient d'un œil inquiet, surtout à cause de la quatrième résolution qui, semble-t-il, prône les principes professés par le journal $l$ A Avenir de Lamennais, dont le procès est pendant à Rome ${ }^{45}$. L'exercice du contrôle qu'on veut laisser à l'évêque est incompatible avec ses multiples occupations. D'ailleurs il ne lui paraît pas bien prouvé qu'il y ait là pour lui un moyen efficace de maîtriser l'opinion publique au profit de l'Eglise. Dans tous les cas, s'il refuse son concours, quel reproche sa conscience pourrait-elle lui en faire? A-t-il jamais pris, lors de sa consécration, quelque engagement relatif à une mesure de ce genre ${ }^{46}$ ?

Devant cette résistance obstinée, $M^{\mathrm{gr}}$ Lartigue décida d'abandonner la partie. Il communiqua sa décision au curé Paquin, son bras droit dans toute cette affaire :

Que reste-t-il à faire à moi, qui dois principalement aux autres l'exemple de l'obéissance à mon Supérieur, que de renoncer le premier

$M^{\mathrm{gr}}$ Lartigue à $\mathrm{M}^{\mathrm{gr}}$ Panet, 20 fév. 1832 (AAM, registre des lettres 6:230).

Cette quatrième résolution se lit comme suit : "Que pour rencontrer ces intérêts réciproques du peuple et du clergé, ce journal ne doit pas être basé sur une théologie étroite qui ne puisse marcher qu'en s'attachant au char de l'arbitraire, mais qui suive les principes de cette saine et haute politique que professent des hommes éclairés et éminemment catholiques, chez les nations où prévaut le besoin indestructible de la liberté constitutionnelle de l'enseignement, de la pensée, de la parole, de la propriété et de la personne, en tant que toutes ces libertés concourent au bien général de la grande famille de l'Etat. 》 $\mathrm{M}^{\mathrm{g} . r}$ Panet à $\mathrm{M}^{\mathrm{gr}}$ Lartigue, 6 mars 1832 (AAQ, registre des lettres 15:34). 
à un projet pour lequel il montre tant d'opposition, ainsi que le Coadjuteur qui lui succèdera sous peu? Je vous déclare donc que je ne me mêlerai plus de cette affaire, jusqu'à ce que de nouvelles circonstances m'autorisent à en reprendre le projet... J'ai fait tout ce qui était en mon pouvoir pour la faire réussir; mais convaincu qu'elle ne saurait avoir de succès sans l'initiative ou du moins l'approbation de l'évêque diocésain, je me lave les mains par rapport aux conséquences, car ma conscience me rend témoignage que $\mathrm{j}$ 'ai fait tout ce qui dépendait de moi $\mathbf{4 7}^{7}$.

$M^{\mathrm{gr}}$ Lartigue ne se priva pas pour autant de dire sa façon de penser à $\mathbf{M}^{\mathrm{gr}}$ Panet. Il lui écrivit donc :

Dieu me garde d'agir contre ce que vous jugez à propos d'établir ou non dans votre diocèse! Vous ne voulez point d'un Journal ecclésiastique, malgré mes représentations réitérées et pressantes; et en conséquence j'ai écrit à ceux qui favorisent la mesure que je ne m'en mêlerais plus en rien et qu'ils ne comptent plus sur mon aide. Cependant je dois vous dire, en finissant, tout ce que j'en pense. Il ne faut pas se créer de fantômes, comme vous faisiez dans votre lettre du 6 , pour les combattre ensuite. Je ne vous proposais pas un journal calqué sur la quatrième ou les autres résolutions de Ste-Geneviève, mais un journal dont le clergé ferait les premiers frais, et qui serait ensuite sous la disposition et propriété absolue de vous et de vos successeurs, pour le diriger et faire marcher entièrement selon votre bon plaisir.

Je regardais et regarde encore ce moyen comme indispensablement nécessaire à la Religion dans les circonstances où nous sommes; ce qui n'est pas difficile à prouver quand on considère l'allure de notre siècle, où l'Evêque ne doit pas se contenter de prêcher dans sa cathédrale, mais établir sa chaire dans un bon papier public, d'où il puisse faire entendre sa voix d'un bout à l'autre de son diocèse pour le maintien de la saine doctrine : en sorte qu'il vaudrait mieux laisser une cure ou quelque autre poste vacants, pour employer un prêtre propre à la rédaction de cet ouvrage sous l'autorité de l'Evêque. Vous n'avez pas promis, il est vrai, à votre consécration, de vous mettre à la tête d'un journal; mais vous avez fait serment de poursuivre toutes les erreurs, de la meilleure manière que vous en seriez capable; et je n'en connais pas qui fût plus efficace en ce moment. Une foule de prêtres, et même de laïcs bien intentionnés désirent cette mesure, comme importante et nécessaire; et il n'aurait pas été difficile de faire 'partir d'ici cet ouvrage avec succès. Mais je n'ai pas voulu que le clergé eût l'air d'être divisé sur une démarche aussi publique : ce qui aurait paru tel, l'évêque diocésain et son coadjuteur n'étant pas de la partie. Quoi qu'il en soit, après tout ce que j'ai fait pour y parvenir, je sens ma conscience déchargée de ce fardeau.

prédiction :

La lettre de $\mathrm{M}^{\mathrm{gr}}$ Lartigue se terminait par cette inquiétante

Je crois pourtant comme presque certain qu'un pareil journal paraîtra quelque jour sans l'avis des supérieurs, conduit peut-être par des prêtres à tête chaude, ou par des hommes de parti et de cabale, capables de s'élever contre les Evêques et de les combattre avec une arme aussi puissante que celle de la presse. Mais alors il ne sera plus temps pour ceux-ci de se ressaisir d'une puissance qu'ils auraient maintenue, s'ils ne l'eussent pas refusée a principio; et je crains beaucoup que vos successeurs n'ayent à le regretter amèrement 48 .

Sur quoi $\mathrm{M}^{\mathrm{gr}}$ Lartigue fondait-il ses prévisions? Redoutait-il l'apparition de quelque nouveau Chaboillez, ou faisait-il simplement

$47 \mathrm{M}^{\mathrm{gr}}$ Lartigue à M. Paquin, 17 mars 1832 (AAM, registre des lettres 6:254).

$48 M^{\text {gr }}$ Lartigue à $M^{\text {gr }}$ Panet, 20 mars 1832 (AAM, registre des lettres 6:257). 
allusion à une décision des prêtres de son district de poursuivre l'exécution du projet de journal en se passant de l'approbation épiscopale? Dans ce dernier cas, aura-t-il prévenu ces prêtres de ne pas négliger cette approbation? Toujours est-il que, deux mois plus tard, sept de ces prêtres - à peu près les mêmes qui avaient passé les résolutions de Sainte-Geneviève - s'adressaient à $\mathrm{M}^{\mathrm{gr}}$ Panet pour lui demander de patronner le journal ou de cesser son opposition à l'entreprise. L'occasion leur paraissait des plus favorable.

Nous voici rendus à une époque où deux partis sont en présence l'un de l'autre et se détachent visiblement des intérêts nationaux pour s'occuper des leurs propres en se combattant. Or il est évident que de ces factions extrêmes il doit nécessairement surgir un parti modéré et national qui, rejetant les prétentions révolutionnaires de l'un et l'esprit de domination de l'autre, se groupera auprès de notre constitution et de notre ordre social. Pourquoi le clergé ne saisirait-il pas cette occasion opportune de susciter ce parti loyal et constitutionnel ? Pourquoi ne tenterait-il pas au moins cet appel à l'ordre et aux lois? Un journal indépendant et modéré, parlant le langage de la vérité et de l'intérêt national, ferait certainement époque. Cette idée est si fortement sentie par tous les amis du pays, qu'on assure qu'il y a un fonds très considérable à Montréal tout prêt à l'établissement d'une presse sur les principes que nous venons d'énoncer. Tous les regards se portent sur Votre Grandeur et l'on attend qu'elle en accepte le patronage, ou qu'elle cesse son opposition à cette entreprise ${ }^{49}$.

$\mathrm{M}^{\mathrm{gr}}$ Panet aurait pu se donner le malin plaisir de relever l'opposition entre cette proposition et la cinquième des résolutions de SainteGeneviève affirmant que le journal ne serait l'écho d'aucun parti : il ne daigna même pas répondre. Et ainsi prit fin cette tentative de fondation d'un journal ecclésiastique.

Huit ans plus tard, en 1840, aussitôt après son accession au siège de Montréal, $\mathrm{M}^{\mathrm{gr}}$ Bourget fondait les Mélanges Religieux, réalisant ainsi le rêve si cher à $\mathrm{M}^{\mathrm{gr}}$ Lartigue, dont il avait été le secrétaire puis le coadjuteur. Il voulut avoir la collaboration de Québec. Il lui fut répondu, comme auparavant à $\mathbf{M}^{\mathrm{gr}}$ Lartigue, qu'on craignait que ce journal n'excitât la colère des ennemis du clergé. Mais il pouvait se passer de l'agrément de Québec, car entre-temps Montréal avait été érigé en diocèse indépendant de celui de Québec.

Thomas-M. Charland, o.p.

49 Lettre de plusieurs curés du district de Montréal à $\mathrm{M}^{\mathrm{gr}}$ Panet, 24 mai 1832 (AAQ, DQ, II-114). 